\title{
Automated Inspection of Solder Paste by Directional LED Lighting
}

\author{
Grantham K.H. Pang and Ming-Hei Chu \\ Department of Electrical and Electronic Engineering, \\ Industrial Automation Research Laboratory, \\ The University of Hong Kong, Pokfulam Road, Hong Kong
} gpang@eee.hku.hk

\begin{abstract}
Screen printing of solder paste is often the very first step in an SMT line in the manufacturing of PCBs. Conventional inspection method would depend on an image acquired from a camera mounted from the top. This 2D inspection of solder paste based on images is fast but is limited to defect such as bridge or no solder. Defects related to the volume of the printed solder paste or unevenness of the paste cannot be treated from a top image. The proposed method of this paper would involve the use of special directional side lighting. A sequence of four images is acquired and image processing is carried out for the edge information of the printed solder paste. A fuzzy system is developed to give a score on the quality of the solder paste. The proposed method can handle other types of defects that cannot be treated by conventional top light images.
\end{abstract}

Index Terms - Process automation, surface mount technology manufacturing, solder paste printing inspection, fuzzy system.

\section{INTRODUCTION}

During the manufacturing of printed circuit board (PCB), the very first stage is the deposit of solder paste pads onto the PCBs. The height of the pads can be obtained based on laser triangulation. However, the inspection speed is slow and very often cannot meet the requirement of an in-line process. Also, a laser scanning system would cost over USD 400 and is very expensive. But the inspection at an early stage would ensure that the specified amount of paste is deposited correctly on the pads. With the right amount of paste, it allows for a greater margin of error in the subsequent component placement and reflow processes.

From some study, it has been found that almost $40 \%$ of defects can be due to poor solder paste printing. Such defects include bridge, bad joints/connection after reflow due to insufficient solder, off registration and excess solder. In fact, the volume of solder paste also affects the quality of the final product. Low solder paste volumes can produce solder joints that pass electrical test but have low mechanical strength and high failure rates. It must be mentioned that not all the end defects are due to the solder paste printing process. However, early defect detection can eliminate many subsequent defects and improve the yield. Also, if defects can be detected immediately after solder paste printing, the repair cost would be the lowest.

The inspection of solder paste using laser is expensive. In laser triangulation, a spot of light from a laser diode is pointed onto the surface of the object under inspection. The reflected laser enters into the window of a light sensitive digital detector array. The height of a point of the object can then be found by triangulation. In practice, a line of laser is projected instead of a laser spot. When the projecting device moves across the object, a camera is used to capture a number of images at even time interval. If high resolution is required, then more images (and also measurement time) are required, which is a time consuming method. This technique involves high-cost machine and not used in a typical production line.

Solder paste inspection in an SMT line usually involves the acquisition of a color image of the printed solder paste blocks under top lighting. Defects such as bridge would appear as a connection between neighboring blocks. No solder would also appear well for the color properties of an area without solder should be quite different from the solder paste. However, defects related to insufficient solder, printed volume or the evenness of the solder paste would not be detected. This paper aims to address on these issues and develops a new method based on the use of directional lighting. A sequence of images are acquired with the use of side light from different directions. Special image processing of the images would highlight the edges, and these edge information would help to detect for the other defects.

\section{REVIEW OF ADVANCED SOLDER PASTE INSPECTION METHODS}

The method for solder paste inspection has been based on constructing the $3 \mathrm{D}$ surface model. The approaches include the followings:

- Laser triangulation method,

- Shape From Shading (SFS) method,

- Phase Profilometry method using structured light source

- Reconstruction by Neural Network. 
The laser method aims to obtain the height of the solder paste by measuring point-by-point or line-by-line the height data by a laser head sensor. The calculation is based on triangulation and the height data of the solder paste is then used for the reconstruction of the 3D profile. Many commercial systems [1] have been developed based on this approach. Accurate measurement can be obtained. However, such laser scan systems are typically very expensive and have low inspection speed.

The first shape-from-shading (SFS) technique was developed by Horn in the early 1970s [2]. The technique is based on recovering the shape from a gradual variation of shading in the image. In SFS, the aim is to recover the light source and the surface shape at each pixel in the image. Zhang et al. [3] have examined other approaches that have emerged and compared their performance. It has been found that none of the six well-known SFS algorithms compared has consistent performance for all test images. They all work well for certain images, but perform poorly for others. Also, the processing time typically required by the SFS algorithms is quite long.

In phase profilometry, a structured light pattern (usually grayscale fringes) is projected onto the object and viewed by a camera. The imaged pattern is phase-modulated according to the topography of the object. The extraction of the phase information enables the reconstruction of the 3D shape of the object [4]. The Fourier transform profilometry [5] relies on processing the image in the frequency domain. Another method relies on processing the image in the real signal domain [6]. In [7], a binary light pattern, Ronchi Pattern, was proposed instead of the traditional grayscale fringe pattern. The Ronchi Pattern can save the space of the light sources and the binary pattern can help to avoid issues like image saturation and specular nature of the imaged surface. A distinct binary code was given to each surface point through a number of physical shifting of the fringe grating and the image data. The height data is then obtained based on correspondence establishment between fringe grating plane and image plane. One drawback of the work is that only the height data at the edge points in the image data can be constructed. The 3D information of non-edge points have to be interpolated from the edge points.

On 3D reconstruction by neural network, an ANN automatic optical inspection model is proposed in [8]. The input nodes of the ANN model consist of the image features that are captured from images using different light sources. The output nodes are the heights of the corresponding image pixel areas. The training patterns of the model use the laser $3 \mathrm{D}$ inspection results. It is claimed that the estimated 3D surface model achieves $90 \%$ accuracy on average.

In another method [9-11], researchers have used neural network to solve problem in SFS, which is then used for 3D shape reconstruction. Wei et al. [9] have used a multilayer neural network approach to tackle the SFS problem. Cho and Chow [10-11] have developed more sophisticated ANN models. In [11], new neural-based reflectance models are presented. The feedforward neural network (FNN) model is able to generalize the diffuse term and the radial-basis function (RBF) model is able to generalize the specular term. Also, a hybrid structure of FNN-based model and RBF-based model is presented because most real surfaces are neither Lambertian models nor ideally specular models.

\section{PROPOSED METHOD FOR SOLDER PASTE INSPECTION}

The proposed method would involve the acquisition of images with directional side lighting. Figure 1 is the top light image of some solder paste blocks. Figure 2 shows the images of the blocks in Figure 1 under directional side lighting from top, bottom, left and right sides.

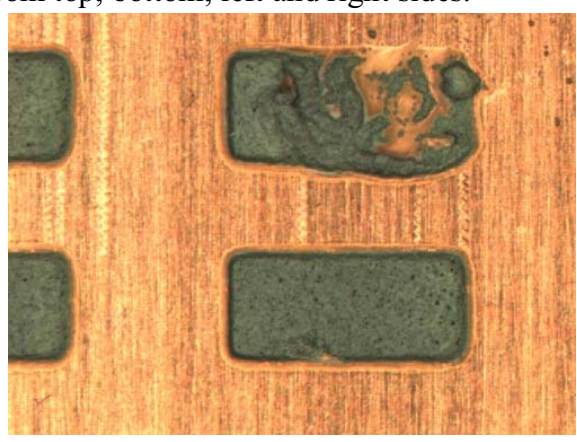

Fig. 1 Top light image of solder paste blocks
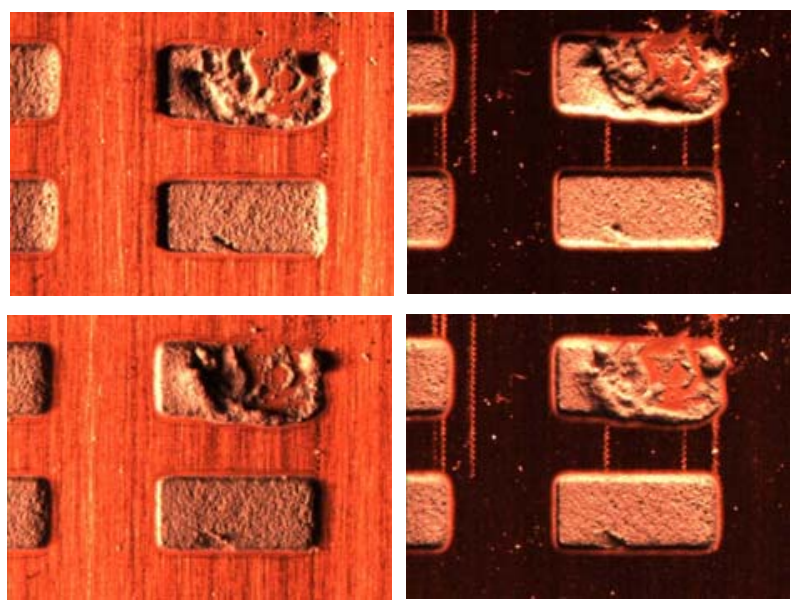

Fig. 2 Side light images of solder paste blocks

The acquired side light images are processed to extract edge area of the solder paste blocks. The processed images of solder paste blocks are shown in Figure 3. It can be observed that a normal solder paste block would give the desirable features on the edges of the four sides. For the defective solder paste block, the edge information of the four sides is very poor. Together with the mask information of the block using the top lighting image, we can know that the 
solder paste block contains defects. With further processing of the edge pixels, a quality score may be computed. Figure 4 contains the block diagram of the proposed method.
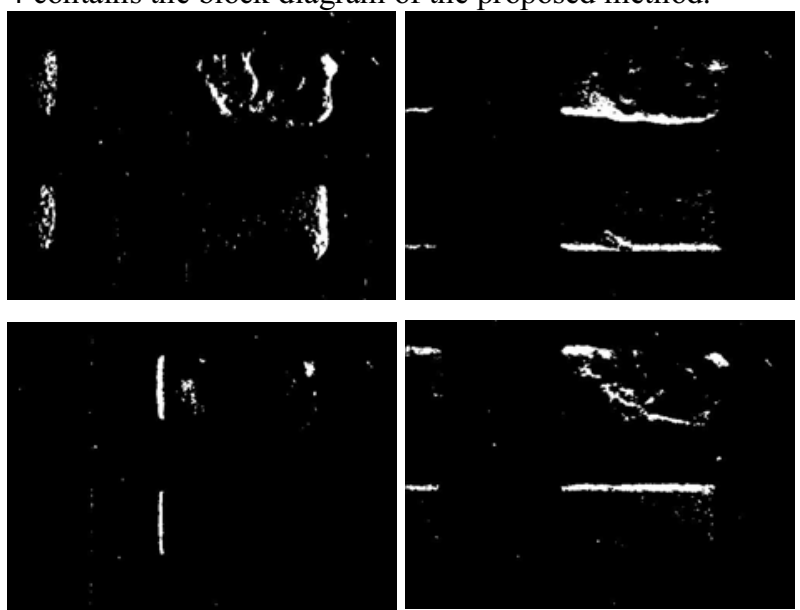

Fig. 3 Processed side light images of solder paste

Solder Paste Block

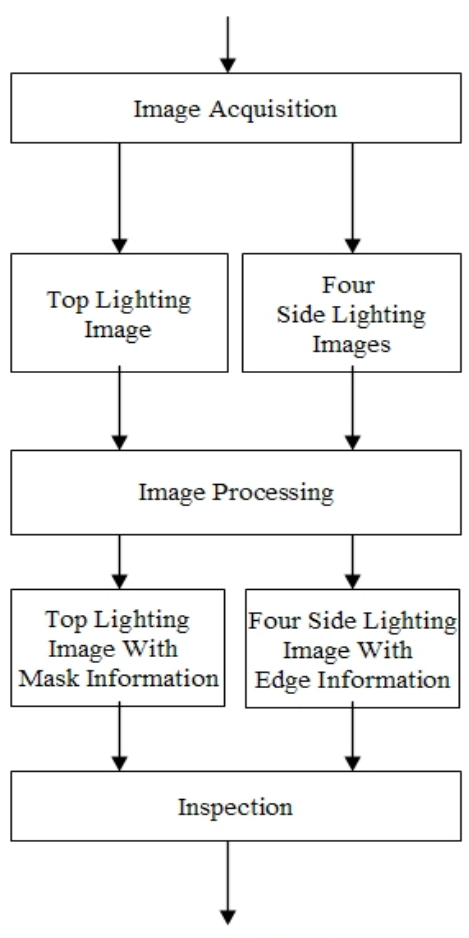

Inspection Result

Fig. 4 Block diagram of the proposed method

\section{Image Processing Module}

The Industrial Automation Laboratory has developed an AOI machine for $2 \mathrm{D}$ inspection of solder paste The current machine is composed of three linear actuators (two for the $\mathrm{Y}$-axis and one for the $\mathrm{X}$-axis) and is essentially a high accuracy X-Y table mounted with an RGB 1280x960-pixel
CCD camera. The position accuracy achieves $0.04 \mathrm{~mm}$. With a field-of-view (FOV) of $23 \mathrm{~mm} \times 17.25 \mathrm{~mm}$, the resolution is 1415 dpi. Photo of the hardware of the developed inspection machine in the Industrial Automation Research Laboratory of the Dept. of Electrical and Electronic Engg. of The University of Hong Kong is given below.

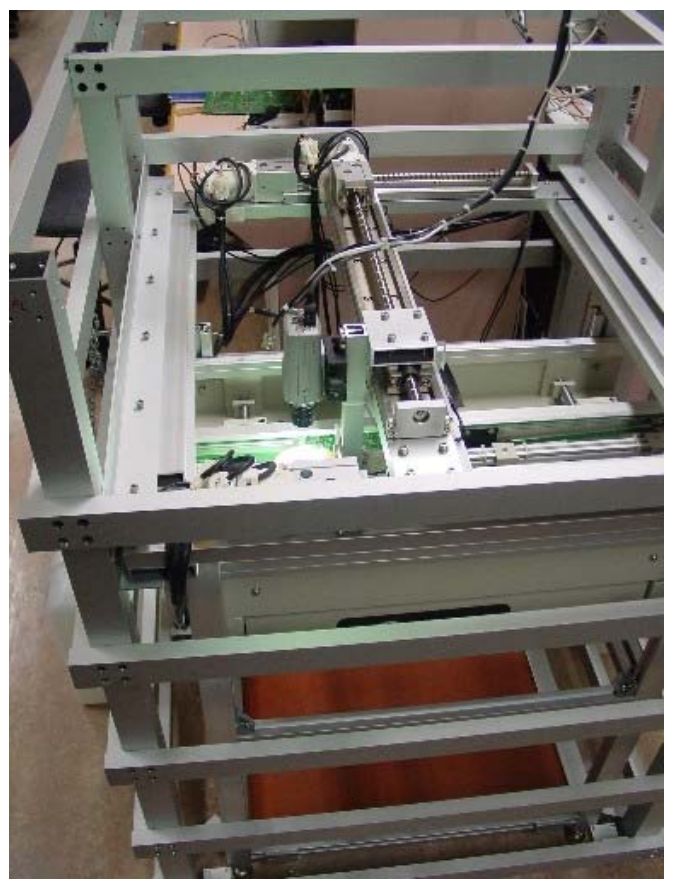

Fig. 5 Developed Inspection Machine

Figure 6, 7 and 8 shows an example of results of a $200 \mu \mathrm{m}$ thick solder paste blocks after image processing.

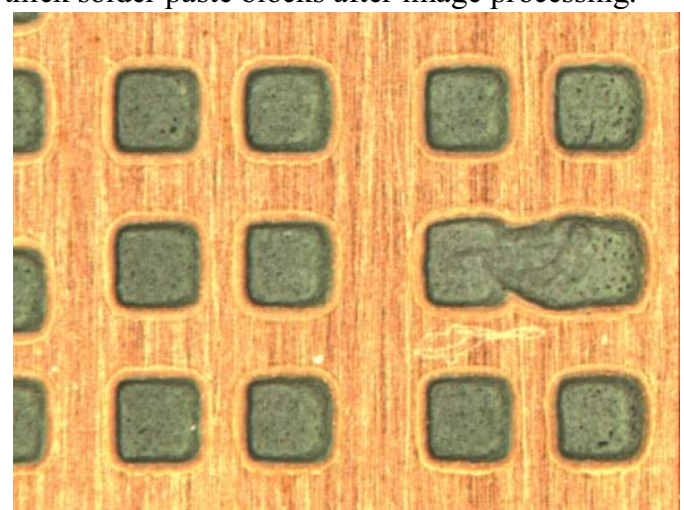

Fig. 6 Top light images of $200 \mu \mathrm{m}$ thick solder paste blocks 

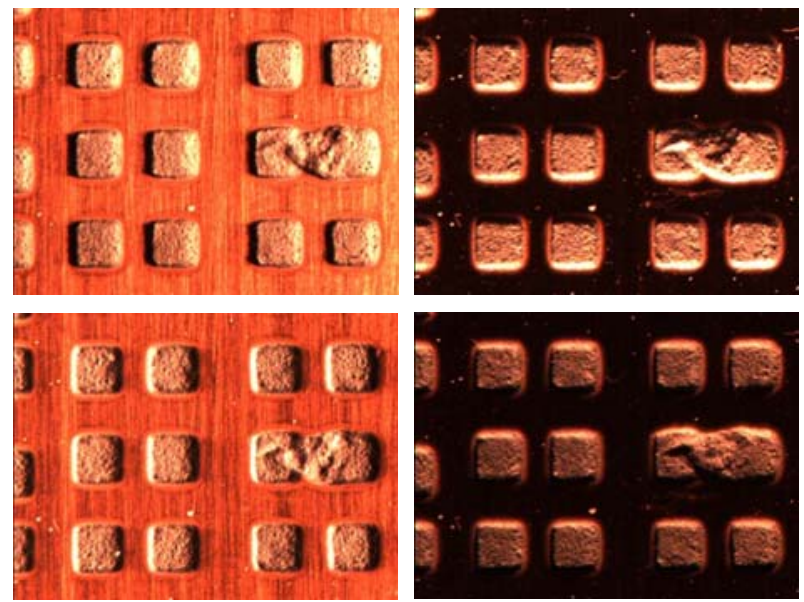

Fig. 7 Side light images of $200 \mu \mathrm{m}$ thick solder paste blocks
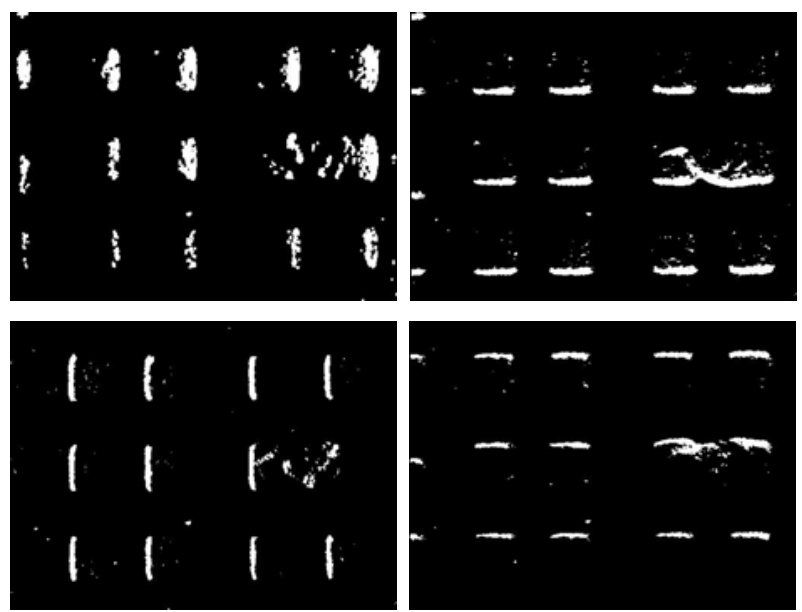

Fig. 8 Processed Side light images of $200 \mu \mathrm{m}$ thick solder paste blocks

\section{FUZZY SYSTEM FOR SOLDER PASTE QUALITY SCORING}

Using the processed solder paste images from the Image Acquisition and Processing Module, the inspection involves a judgment on the quality of the solder paste block based on a set of fuzzy rules. A fuzzy system is first setup based on a set of fuzzy rules for solder paste inspection. The system extracts features from the processed solder paste images and uses the extracted features for judgment. A quality score is then calculated from the extracted features.

The initial fuzzy system setup is based on the reference solder paste samples. Neural-fuzzy adaptation can be carried out on the fuzzy system to fine tune its behavior so that it can meet the users' expected inspection results. To carry out adaptation, users of the system are required to provide a set of solder paste training samples with expected quality scores. Then standard neural-fuzzy training algorithm is used to train the system using the provided training samples. The trained fuzzy system will work closer to the users' expectation by giving scores close to the users' expected scores. This feature means the fuzzy system can adapt to inspect different types of solder paste blocks.

The inspection step consists of two modules as shown in Figure 9. The first module, Module A, is the fuzzy system for quality score calculation. It calculates the quality score of the solder paste based on a set of fuzzy rules from the input solder paste features. The second module, Module B, is the fuzzy-neural network for fine tuning the performance of the Module A. The tuning process is carried out by adjusting the membership functions of the fuzzy system.

In general, a typical non-defective solder paste block consists of a central region and four edge regions: North, South, East and West Edges. The central region is the region bounded by the four edges. The position, size and shape of these regions reflect the physical shape of the solder paste block and, hence, the quality of the solder paste block. From the study of a large number of defective and non-defective solder paste samples, it is found that non-defective solder paste samples with similar thicknesses gives similar positions and sizes of the edge regions and central regions. For defective samples, any defects in the central region will appear as blue or red color clusters. To determine the quality of the solder paste blocks, the above regions need to be analyzed to give the final quality score.

For edge regions, the shape of non-defective solder paste blocks should not have large deviation in the shape and size. As the edge thickness and the edge area reflects the shape of the edge regions, the average edge thickness and edge surface area for each edge are selected as input features of the fuzzy system. Also, the thickness deviations of the edges are useful for judging the quality of the edge regions. In addition, discontinuities in the edge regions mean that defects exist in the edges. In addition, the connectivity of the edge region needs to be studied. As a result, for edge regions, edge thickness, edge thickness deviation, edge surface area and edge connectivity are chosen as the input features of the fuzzy system.

For the whole solder paste block, the position and the surface area are closely related to the printing quality. Shifting of the printing position is one of the major defects in solder paste printing. Also, the surface area of the solder paste block can help to inspect whether there is sufficient solder paste is printed. As a result, solder paste surface area and block shifting are included as input features of the fuzzy system.

After an analysis of the solder paste shape, many features of the solder paste block are extracted. Examples of their 
descriptions are given as follows:

- Feature on Edge Thickness : Obtained by calculating the average width of all the rows of blue pixels in the Edge Region

- Feature on Edge Thickness Deviation : Obtained by calculating the standard deviation of the Edge Thickness

- Feature on West Edge pixels' connectivity : Obtained by counting the number of gaps vertically in the Edge Region

- Feature on West Edge Region Surface Area : Obtained by calculating the number of red pixels in the Edge Region

These features are obtained from processed side light images of the solder paste blocks. Next, we discuss on the developed fuzzy rules for the inspection of the solder paste. Examples of two fuzzy rules are shown below as an illustration:

- IF West Edge Thickness is GOOD and East Edge Thickness is GOOD and North Edge Thickness is GOOD and South Edge Thickness is GOOD, THEN quality score is HIGH.

- IF West Edge Thickness Deviation is BAD or East Edge Thickness Deviation is BAD or North Edge Thickness Deviation is BAD or South Edge Thickness Deviation is BAD, THEN quality score is LOW.

The proposed fuzzy system has been evaluated with solder paste blocks of different shapes and thicknesses. In the evaluation of square-shaped solder paste blocks, the proposed fuzzy system is tested against solder paste blocks with different thicknesses. The processing time for 100 samples is about 0.2 second. Figure 10 shows that it can classify solder paste blocks with different thicknesses into different categories.

The fuzzy system was trained to identify for solder paste blocks of $150 \mu \mathrm{m}$ and it has successfully distinguishes defective blocks from the testing samples. In this experiment, a set of 69 solder pastes samples have been used to evaluate the performance of the fuzzy system. The score distribution graph shows that all the defective solder paste samples can be successfully identified (those below a score of 0.28 ). In addition, the neural-fuzzy training can help to fine-tune the behavior of the fuzzy system according the users' preferences. Experiment results show that the fine-tuned fuzzy system gives a high accuracy in the defect detection of solder paste blocks.

\section{CONCLUSIONS}

The proposed method of this paper involves the use of special directional side lighting on the solder paste blocks. A sequence of four images is acquired and image processing is carried out for the edge information of the printed solder paste. The proposed method can handle other types of defect that cannot be treated by conventional top lighting images. Results from an experiment have shown that the proposed fuzzy system can be used for detecting defects in the solder paste blocks. In addition, it can detect any incorrect printing thicknesses during the SMT process. With proper training and setup, the fuzzy system can provide high detection accuracy.

\section{REFERENCES}

[1] Jeffrey Rupert, Doreen Tan and Pat Pilon, "3D Inspection for the measurement of solder paste deposits", APEX 2000, March 14-16, 2000.

[2] B.K.P. Horn, "Shape from Shading: A Method for Obtaining the Shape of a smooth Opaque Object from One View", Ph.D. thesis, Massachusetts Instituteof Technology, 1970.

[3] Ruo Zhang; Ping-Sing Tsai; Cryer, J.E.; Shah M. "Shape From Shading: A Survey", IEEE Transactions on Pattern Analysis and Machine Intelligence Volume 21, Issue 8, pp. $690-706$, Aug. 1999.

[4] Luigi Di Stefano and Frank Boland, "A new phase extraction algorithm for phase profilometry", Machine Vision and Applications, Volume 10, Number 4, pp. 188 - 200, December 1997.

[5] M. Takeda and K. Mutoh, "Fourier transform profilometry for the automatic measurement of 3-D object shapes", Applied Optics, 22 (24), pp. 3977-3982, 1982.

[6] S. Tang and Y. Hung, "Fast profilometer for the automatic measurement of 3-D object shapes", Applied Optics, 29 (20), pp 3012-3018, 1990.

[7] Jun Cheng, Ronald Chung, Edmund Y. Lam, Kenneth S.M. Fung, Fan Wang, and W.H. Leung, "Three-dimensional reconstruction of wafer solder bumps using binary pattern projection," in Machine Vision Applications in Industrial Inspection XIII, vol. 5679 of Proceedings of the SPIE, pp. 44-52, January 2005.

[8] Fang-Chung Yang, Chung-Hsien Kuo, Jein-Jong Wing, Ching-Kun Yang, "Reconstructing the 3D solder paste surface model using image processing and artificial neural network", 2004 IEEE International Conference on Systems, Man and Cybernetics, Volume 3, 10-13, pp. 3051 - 3056, Oct. 2004.

[9] G.Q. Wei and G. Hirzinger, "Learning shape from shading by a multilayer network", IEEE Trans. Neural Networks, Vol. 17, pp. 985-995, 1996

[10] S. Y. Cho and T. W. S. Chow, "Shape recovery from shading by a new neural-based reflectance model", IEEE Trans. Neural Networks, Vol. 10, pp. 1536-1541, 1999.

[11] S.Y. Cho and T.W. S. Chow, "Neural Computation Approach for Developing a 3-D Shape Reconstruction Model", IEEE Transactions on Neural Network, Vol. 12, No. 5, pp. 1204-1214, September 2001. 


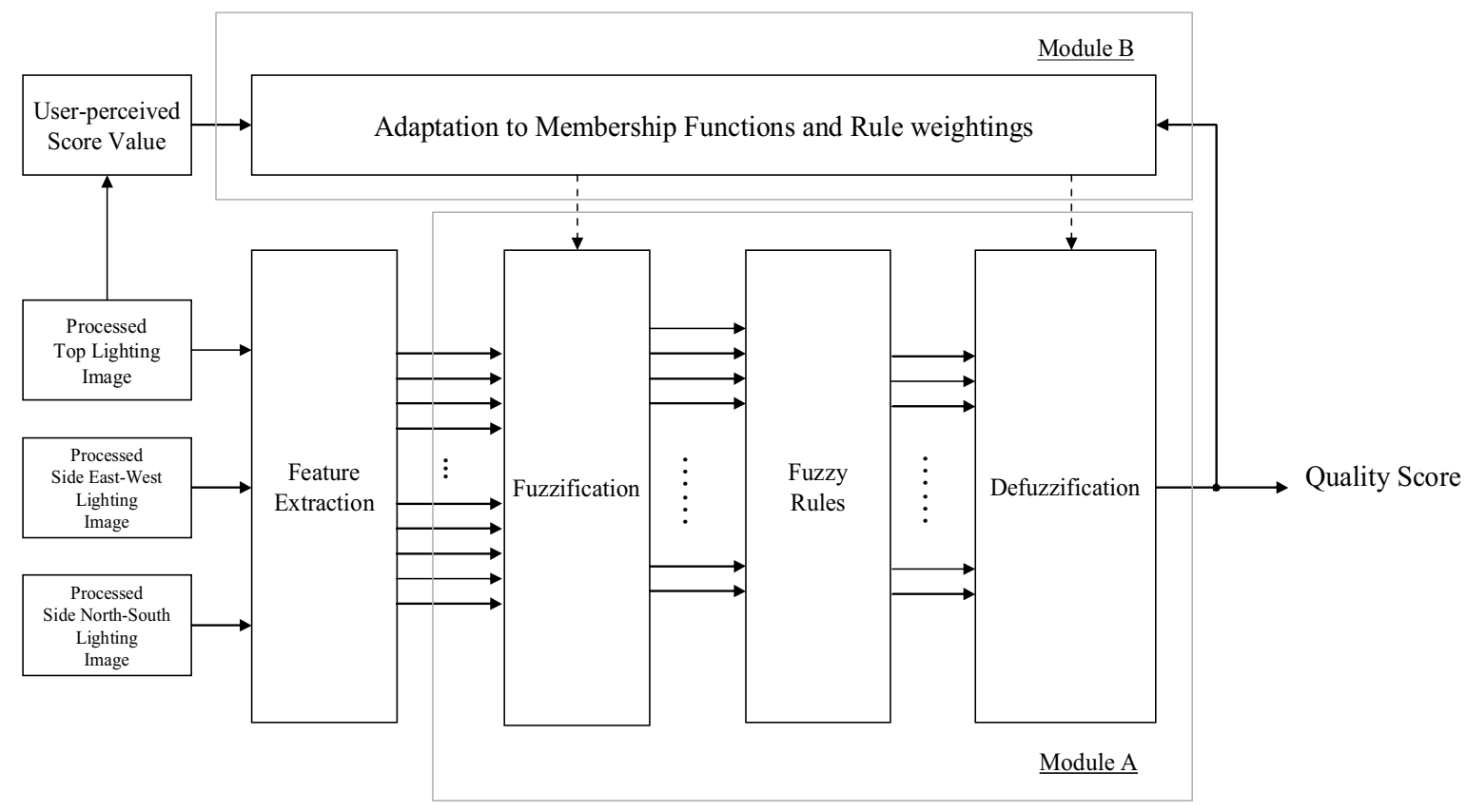

Fig. 9 Block diagram of fuzzy system for inspection of solder paste quality

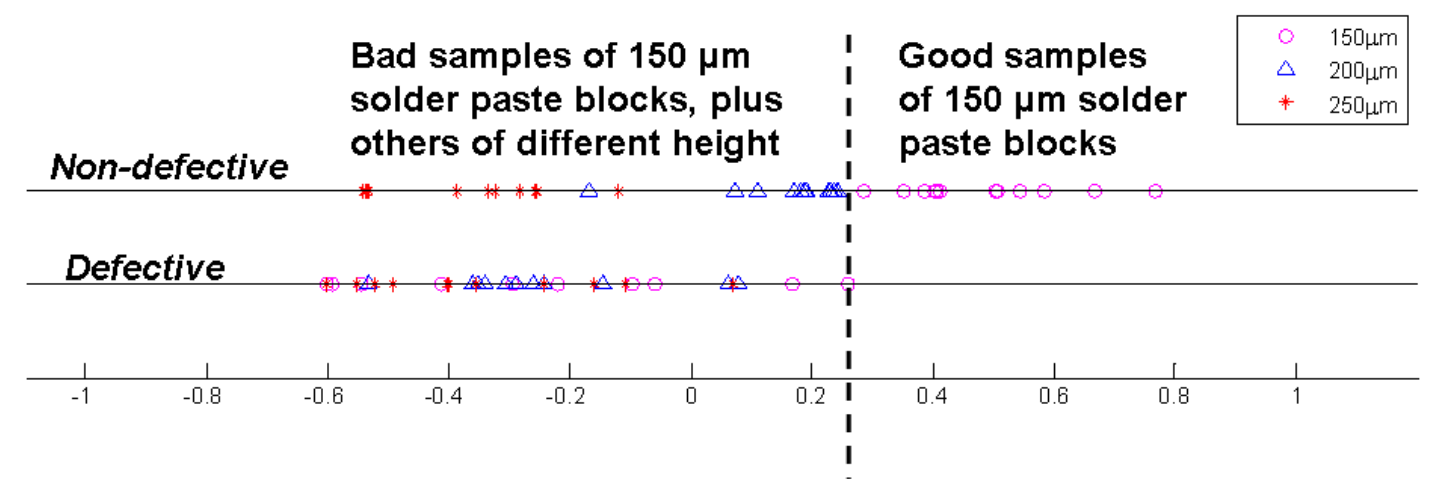

Fig. 10 Score distribution graph of testing solder paste samples 